\title{
Predictive model using prostate MRI findings can predict candidates for nerve sparing radical prostatectomy among low- intermediate risk prostate cancer patients
}

\author{
Gang Song ${ }^{1,2,3 \#}$, Mingjian Ruan ${ }^{1,2,3 \#}$, He Wang ${ }^{4}$, Zhiyong Lin ${ }^{4}$, Xiaoying Wang ${ }^{4}$, Xueying Li ${ }^{5}$, Peng Li ${ }^{6}$, \\ Yandong Wang ${ }^{1,2,3,7}$, Binyi Zhou ${ }^{1,2,3}$, Xuege Hu ${ }^{1,2,3}$, Hua Liu ${ }^{1,2,3}$, Hao Wang ${ }^{1,2,3}$, Yinglu Guo ${ }^{1,2,3}$ \\ ${ }^{1}$ Department of Urology, Peking University First Hospital, Beijing 100034, China; ${ }^{2}$ Institute of Urology, Peking University, Beijing 100034, China; \\ ${ }^{3}$ National Urological Cancer Center of China, Beijing 100034, China; ${ }^{4}$ Department of Radiology, ${ }^{5}$ Department of Statistics, ${ }^{6}$ Department of \\ Ultrasound, Peking University First Hospital, Beijing 100034, China; ${ }^{7}$ Department of Urology, the People's Hospital of Guizhou Province, Guiyang \\ 550002, China \\ Contributions: (I) Conception and design: G Song, Y Guo; (II) Administrative support: None; (III) Provision of study materials or patients: G Song; \\ (IV) Collection and assembly of data: M Ruan, P Li, Y Wang, B Zhou, X Hu, H Liu, H Wang; (V) Data analysis and interpretation: M Ruan, X Li, \\ G Song; (VI) Manuscript writing: All authors; (VII)Final approval of manuscript: All authors. \\ "These authors contributed equally to this work. \\ Correspondence to: Gang Song. Department of Urology, Peking University First Hospital, Beijing 100034, China. Email: sgbmupaper@163.com.
}

Background: In order to improve postoperative functional outcome, including urinary continence and erectile function, nerve sparing surgery is recommended for patients with clinically localized prostate cancer (PCa). However, due to poor diagnosis accuracy at the preoperative stage, upstaging occurs in a considerable proportion of patients. Multiparametric magnetic resonance imaging (mpMRI) and the Prostate Imaging Reporting and Data System version 2 (PI-RADS v2) have recently shown excellent performance in diagnosis and staging of PCa. The aim of this study was to develop a predictive model based on PI-RADS v2 for postoperative upstaging in patients with low-intermediate risk PCa.

Methods: The medical records of 314 patients with low-intermediate risk PCa [prostate-specific antigen (PSA) level $\leq 20 \mathrm{ng} / \mathrm{mL}$, Gleason score $(\mathrm{GS})<8$, and clinical stage $<\mathrm{T} 3$ ] who underwent preoperative mpMRI and radical prostatectomy in the Department of Urology, Peking University First Hospital between January 2012 and July 2019 were reviewed retrospectively. Clinicopathological characteristics were collected. All MRI reports were done at our institution as part of routine clinical practice before prostate biopsy and there was no re-reporting occurred. Using PI-RADS v2, the mpMRI results were assigned to three groups: "negative", "suspicious", and "positive". Multivariate logistic regression analysis was used to assess factors associated with postoperative pathological upstaging, defined as the presence of pT3 at final pathology. A regression coefficient based model for predicting postoperative upstaging was constructed and internally validated using 1,000 bootstrap resamples. The performance of the model was assessed using the area under the receiver operating characteristic curve (AUC). With the optimal cutoff point the performance of the model was assessed through analysis of sensitivity, specificity, positive predictive value, and negative predictive value.

Results: Upstaging was observed in 119 (37.9\%) patients. The univariate and multivariate analyses revealed that PSA density, biopsy Gleason grade group (GGG), and mpMRI findings were significantly independent predictors for postoperative upstaging (all $\mathrm{P}<0.05$ ). A predictive model showing very favorable calibration characteristics and higher accuracy than the single variables was constructed (AUC $=0.74 ; \mathrm{P}<0.001$ ). At the optimal cutoff point, the model demonstrated a sensitivity and negative predictive value of $87.4 \%$ and $87.0 \%$, respectively.

Conclusions: PI-RADS v2 assessment proved to be one of the most valuable predictors for postoperative upstaging in patients with low-intermediate risk PCa. The predictive model, based on PI-RADS v2 assessment, PSA density, and biopsy GGG, may help to select suitable candidates for nerve sparing radical 
prostatectomy among patients with low-intermediate risk PCa.

Keywords: Prostate cancer (PCa); multiparametric MRI; radical prostatectomy; nerve sparing

Submitted Sep 29, 2019. Accepted for publication Jan 05, 2020.

doi: $10.21037 /$ tau.2020.01.28

View this article at: http://dx.doi.org/10.21037/tau.2020.01.28

\section{Introduction}

For the majority of patients with clinically localized prostate cancer $(\mathrm{PCa})$, radical prostatectomy is the standard treatment, providing excellent oncological outcome. However, postsurgical urinary incontinence and erectile dysfunction, the most common postoperative complications, severely affect quality of life of the patients.

Since nerve sparing surgery was introduced, substantial improvement has been made in functional outcome in terms of urinary continence and erectile function $(1,2)$. However, appropriate candidate selection for nerve sparing surgery is essential to balance the risks of oncologic safety and functional outcomes. According to American Urological Association guidelines, nerve sparing radical prostatectomy (NSRP) is recommended for patients with localized disease (3). Current European Association of Urology guidelines recommend not to preserve the neurovascular bundles in case of local advanced PCa or any biopsy Gleason score (GS) $>7$ (4).

Preoperative clinical staging based on digital rectal examination and prostate biopsy has limited accuracy, with understaging of locally extensive diseases in $30.4 \%$ to $37.2 \%$ of cases $(5,6)$. In one study, $39.8 \%$ of patients who underwent NSRP were upstaged, leading to a higher rate of positive surgical margin and worse prognosis (7). NSRP was not suitable for these patients. These proportions confirmed the need for predicting the presence of upstaging at final pathology. Several predictive models have been constructed in order to predict postoperative upstaging $(6,8,9)$. However, no models currently integrate the PI-RADS score to predict upstaging. Multiparametric magnetic resonance imaging (mpMRI) and the Prostate Imaging Reporting and Data System version 2 (PI-RADS v2) (10) currently play an important role in diagnosis and staging of $\mathrm{PCa}$.

Therefore, the purpose of this study was to construct a new PI-RADS v2 based model for predicting postoperative upstaging in patients with clinically localized PCa. The developed predictive model may guide urologists in selecting appropriate candidates for nerve sparing operation.

\section{Methods}

\section{Patient population}

A total of 639 patients, who underwent preoperative mpMRI followed by radical prostatectomy for clinically localized PCa at our institution between January 2012 and July 2019, were reviewed retrospectively. Of the 639 patients, 401 were classified as low-intermediate risk PCa [prostate-specific antigen (PSA) level $\leq 20 \mathrm{ng} / \mathrm{mL}, \mathrm{GS}<8$, and clinical stage < T3] according to the NCCN 2019 Clinical Practice Guidelines in Oncology (11).

In all, 87 patients were excluded based on the following criteria:

(I) prior hormonal therapy or radiotherapy [44];

(II) less than 10 or $>13$ systematic biopsy cores taken [23];

(III) incomplete data [20].

As a result, a total of 314 patients were enrolled in the study. Approval was obtained from the institutional review board.

\section{Clinicopathological characteristics}

Clinicopathological characteristics of the patients, i.e., age, preoperative PSA level, prostate volume (PV) measured by transrectal ultrasound (TRUS), the percentage of positive systematic biopsies which was defined as the proportion of the number of positive systematic cores to the number of total systematic cores, GS following prostate biopsy, and pathologic characteristics of specimens following radical prostatectomy, were collected. PSA density was calculated by dividing the preoperative PSA by the PV. Patients who were classified into the high-risk group were excluded because such patients can hardly be considered as candidates for nerve sparing procedures or other focal therapy.

\section{Biopsy procedure and bistopathology}

All patients underwent 10- to 13-core TRUS guided systematic biopsy at our hospital. In those patients where 
suspicious lesions at any area were found by ultrasonography or mpMRI, two targeted biopsies were performed, but these targeted biopsies were not analyzed.

All biopsy specimens were evaluated by two genitourinary pathologists, in order to determine the cancer stage and the GS in positive cases. The GS for individual TB and SB cores, as well as the overall GS for each patient, was assigned as the combination of the most common Gleason grade and highest Gleason grade. The new GS grading system was used to classify patients into the following five grades: grade group 1, GS 6; grade group 2, GS 3+4=7; grade group 3, GS 4+3=7; grade group 4, GS 4+4=8; and grade group 5, GS 9 and 10 (12). The pathological stage was determined in accordance with the AJCC 2017 TNM classification system. Upstaging was defined as showing p T3 features at final pathology, such as extra-capsular extension, defined as the extension of tumor beyond the boundaries of the prostate gland into periprostatic adipose.

\section{MpMRI}

MRI was performed using a $1.5 \mathrm{~T}$ for $52(16.6 \%)$ patients and 3.0T whole-body system (GE Healthcare, USA) for 262 (83.4\%) with no endorectal coil used. The imaging protocol included axial T1-weighted images of the pelvis and biplanar T2-weighted fast spin-echo images centered on the prostate. In addition, axial diffusion-weighted imaging was performed with b-values of 0,800 , and $1,400 \mathrm{sec} / \mathrm{mm}^{2}$. Dynamic contrast-enhanced images were made following intravenous administration of gadolinium-chelate.

\section{MpMRI interpretation}

MRI images were retrospectively interpreted by one of two experienced radiologists with $>5$ years' experience reading prostate MRIs. Any disagreement in the process of interpretation was resolved by the adjudicating senior radiologist. The probability of tumor was evaluated and scored on a three-point scale based on PI-RADS v2 scoring, where group "negative" (PI-RADS 1-2) = low probability, group "suspicious" (PI-RADS 3) = equivocal, and group "positive" (PI-RADS 4-5) = high or very high probability (10).

\section{Statistical analysis}

The end point of the study was identification of upstaging at postoperative pathology (T3 stage). Descriptive statistics included frequencies and proportions for categorical variables and means, standard deviations, medians, and interquartile ranges (IQR) for continuously coded variables. Univariate and multivariate logistic regression analyses were performed to investigate potential independent predictors of upstaging at final pathology. The factors evaluated for prediction of upstaging at postoperative pathology were age, preoperative PSA level, PV by TRUS, PSA density, percentage of positive systemic biopsies, Gleason grade group (GGG) following prostate biopsy, and PI-RADS v2 score.

The logistic regression model for upstaging at postoperative pathology was constructed, utilizing selected variables based on the results of multivariate logistic regression analysis. Discrimination was measured using the area under the curve (AUC) derived from the receiver operating characteristic (ROC) curves. In addition, 1,000 bootstrap resamples were used for internal validation and to reduce overfit bias. With the optimal cutoff value according to Youden's index, the performance of the model was assessed through analysis of sensitivity, specificity, positive predictive value, and negative predictive value. All analyses were two-sided, with statistical significance set at $\mathrm{P}<0.05$. The statistical analyses were performed using SPSS (version 24.0; SPSS Inc., Chicago, IL, USA) and R (The R Foundation for Statistical Computing, Vienna, Austria).

\section{Results}

The baseline clinical and pathological characteristics of the 314 patients in this cohort are described in Table 1. The median age was 67 (IQR, 62-71) years. The median preoperative PSA was 8.83 (IQR, 6.90-11.80) ng/mL.

Of all 314 patients, $119(37.9 \%)$ presented with pT3 PCa at postoperative pathology, i.e., upstaging. Before operation, 107 cases (34.1\%), 150 cases (47.8\%), and 57 cases (18.2\%) were stratified into GGG 1, 2 and 3, respectively. Of those who were diagnosed with GGG 1 cancer by biopsy, 26 $(24.3 \%)$ were diagnosed with a locally advanced disease at final pathology; this number was $63(42.0 \%)$ for those with GGG 2, and 30 (52.6\%) for those with GGG 3 .

The univariate and multivariate logistic regression analyses in this study cohort are described in Table 2. On univariate analysis, patients presenting upstaging in postoperative pathology showed significantly higher PSA [odds ratio (OR), 1.141; 95\% confidence interval (CI), $1.072-1.215 ; \mathrm{P}<0.001]$ and $\mathrm{PSA}$ density values $(\mathrm{OR}, 51.743$; 95\% CI, 9.553-280.262; $\mathrm{P}<0.001)$, lower free/total $(\mathrm{f} / \mathrm{t})$ 
Table 1 Baseline clinical and pathological characteristics of the study cohort

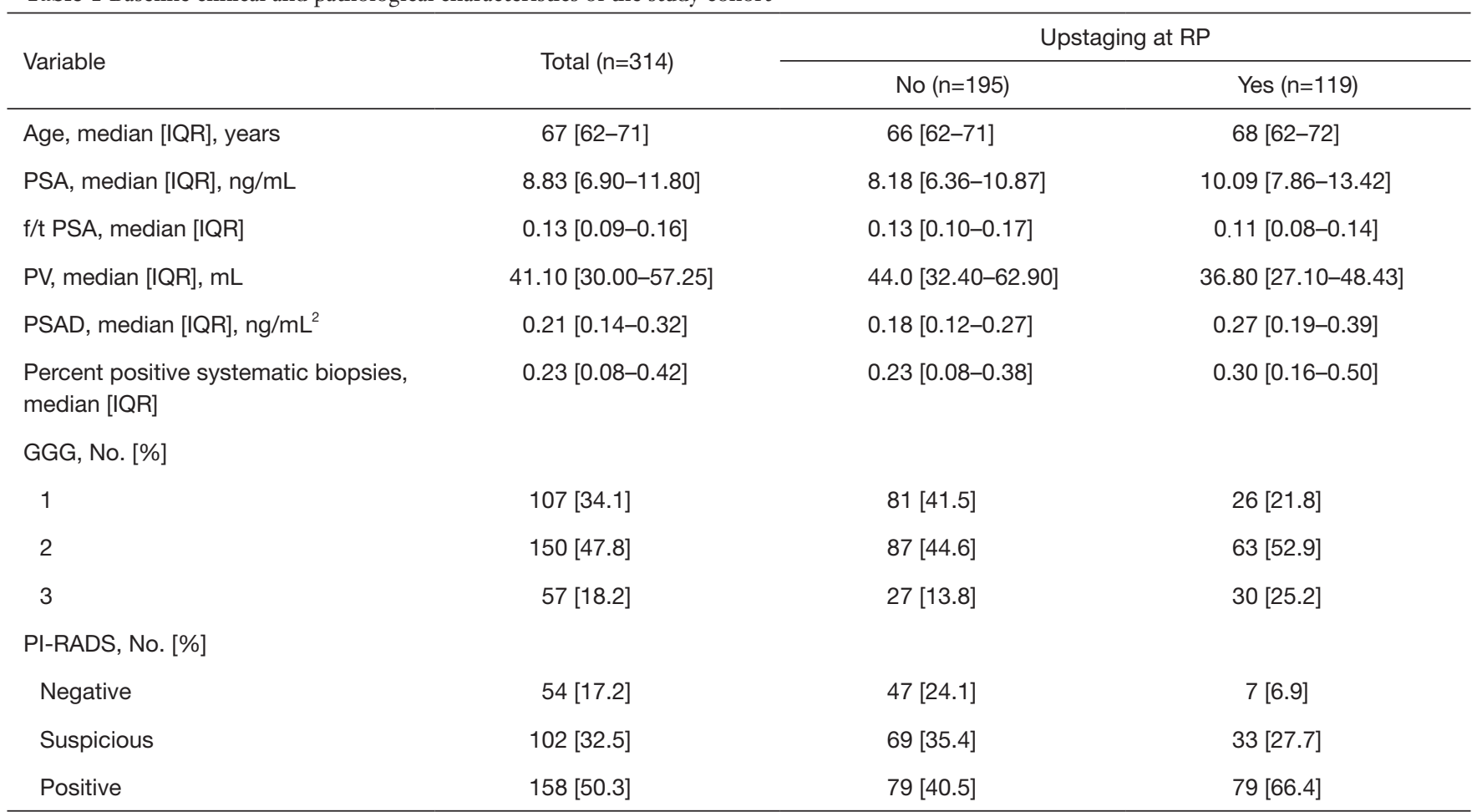

RP, radical prostatectomy; IQR, interquartile range; BMI, body mass index; PSA, prostate-specific antigen; f/t PSA, free/total PSA ratio; PV, prostate volume; PSAD, PSA density; GGG, Gleason grade group; PI-RADS, Prostate Imaging Reporting and Data System.

Table 2 Univariate and multivariate analyses of predictors associated with upstaging prostate cancer

\begin{tabular}{|c|c|c|c|c|}
\hline Variable & Univariate & $P$ value & Multivariate & $P$ value \\
\hline Age (years) & $1.027(0.991-1.064)$ & 0.144 & & \\
\hline PSA (ng/mL) & $1.141(1.072-1.215)$ & $<0.001^{*}$ & & \\
\hline$f / t$ & $0.007(0-0.521)$ & $0.024^{*}$ & & 0.678 \\
\hline $\operatorname{PSAD}\left(\mathrm{ng} / \mathrm{mL}^{2}\right)$ & 51.743 (9.553-280.262) & $<0.001^{*}$ & $26.577(4.657-151.680)$ & $<0.001^{*}$ \\
\hline Percent positive systematic biopsies & 8.267 (2.563-26.662) & $<0.001^{*}$ & & 0.568 \\
\hline GGG & $1.893(1.353-2.650)$ & $<0.001^{*}$ & $1.572(1.095-2.257)$ & $0.014^{*}$ \\
\hline \multicolumn{5}{|l|}{1} \\
\hline \multicolumn{5}{|l|}{2} \\
\hline PI-RADS & $2.401(1.687-3.418)$ & $<0.001^{*}$ & $2.022(1.387-2.947)$ & $<0.001^{*}$ \\
\hline \multicolumn{5}{|l|}{ Negative } \\
\hline \multicolumn{5}{|l|}{ Suspicious } \\
\hline Positive & & & & \\
\hline
\end{tabular}

*, P value with $<0.05$ significance. OR, odds ratios; $\mathrm{Cl}$, confidence interval. 


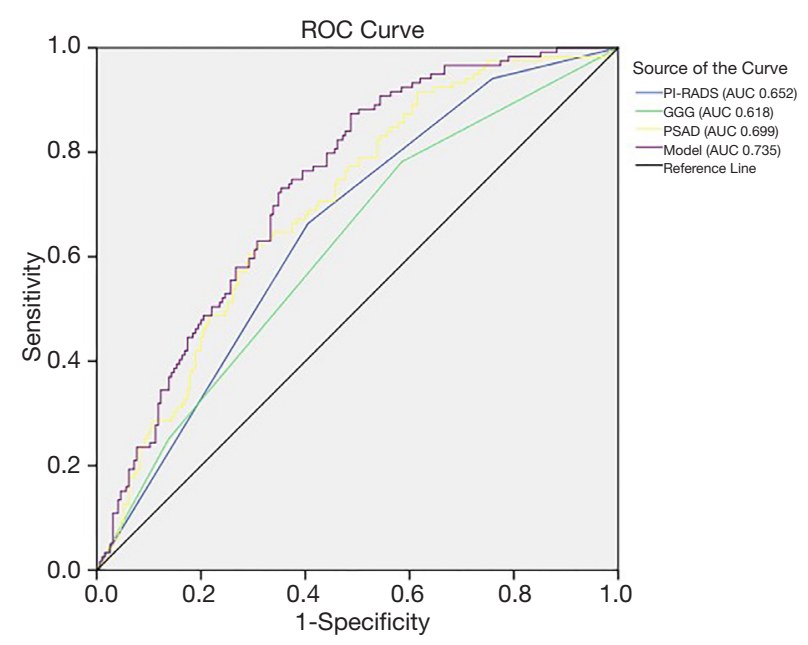

Figure 1 Receiver operating characteristic curves (ROC) for upstaging at final pathology for PSAD, PI-RADS score, GGG and predictive model. GGG, Gleason grade group; PI-RADS, Prostate Imaging Reporting and Data System.

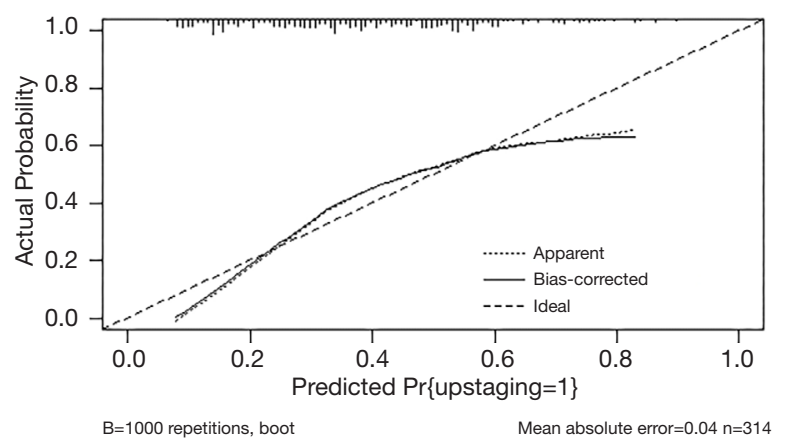

Figure 2 Calibration plot for predicting postoperative upstaging probability.

PSA values (OR, 0.007; 95\% CI, 0-0.521; $\mathrm{P}=0.024)$, smaller prostates (OR, 0.980; 95\% CI, 0.969-0.992; $\mathrm{P}<0.001$ ), a higher percentage of positive systematic biopsies (OR, 8.267; 95\% CI, 2.563-26.662; $\mathrm{P}<0.001$ ), higher GGGs (OR, 1.893; 95\% CI, 1.353-2.650; $\mathrm{P}<0.001$ ), and a higher PIRADS score (OR, 2.401; 95\% CI, 1.687-3.418; $\mathrm{P}<0.001$ ) compared to men without upstaging. No difference in age was observed between patients with or without postoperative upstaging.

The PSA and PV data were excluded from the multivariate analysis to avoid confounding. In the multivariate analysis, PSA density, GGG following biopsy, and PI-RADS score remained significantly associated with postoperative upstaging, suggesting that these variables are independent risk predictors. Of those, the PI-RADS score was the most significant predictor according to the standard regression coefficient. Specifically, patients with high PIRADS scores were at higher risk of upstaging at final pathology.

With application of the coefficients of the logistic function, a predictive model for postoperative upstaging was constructed using selected risk factors, including PSA density, GGG, and PI-RADS score, as follows:

$\operatorname{logit}(\mathrm{P})=\operatorname{In}[(\mathrm{P} /(1-\mathrm{P})]$

$=-3.188+0.704 \times P I-R A D S+0.453 \times G G G+3.280 \times P S A D$.

In this equation, GGG 1, GGG 2, GGG3 were assigned to $0,1,2$, respectively into the equation for calculation. PIRADS outcome like negative, suspicious and positive results were also denoted as $0,1,2$, respectively.

ROC analysis was performed to assess the accuracy of the model, as shown in Figure 1. The predictive model applied for postoperative upstaging yielded an AUC of 0.735, with a $95 \%$ CI of $0.681-0.790(\mathrm{P}<0.001)$, which was better than any single risk factor. At the optimal cutoff predictive value of 0.295 , it showed a sensitivity of $87.4 \%$, a specificity of $51.0 \%$, a positive predictive value of $52.3 \%$, and a negative predictive value of $87.0 \%$. The calibration plot, exploring the relationship between observed and predicted values, before and after bootstrap validation, is depicted in Figure 2.

\section{Discussion}

Distinguishing organ-confined disease from disease with locally advanced PCa is of significance when planning treatment strategies for PCa. Among men with lowintermediate risk PCa before surgery, those presenting $\geq$ pT3a PCa at final pathology should be excluded from the local control cohort (nerve sparing surgery or focal therapy) of $\mathrm{PCa}$, so as to improve the likelihood of achieving negative margins and better prognosis. Moreover, patients with local advanced $\mathrm{PCa}$ will be given more aggressive treatment for the sake of cancer control and avoiding biochemical recurrence.

Current staging relies heavily on mpMRI, especially for patients with local advanced PCa. However, according to a contemporary critical meta-analysis, involving 9,796 patients from 75 different studies, MRI showed a poor sensitivity of 0.61 (95\% CI, 0.54-0.67) for all patients with stage T3 PCa (13), which means a number of patients with local advanced PCa were missed, which may lead to relatively conservative management of PCa. Our results show an upstaging rate of $37.9 \%$ within the entire cohorts, 
which is similar to results from previous studies $(5,6)$. Nevertheless, unlike other authors, we did not consider those with documented loss of prostate capsule and irregularity of the capsule by mpMRI owing to its high positive predictive value. This may be due to the relatively high proportion of patients with biopsy GS 7 in this study. Moreover, a recent study focusing on Korean patients found that nearly $50 \%$ of patients with low risk disease were upstaged postoperatively (14), a number we cannot neglect.

Several models for postoperative upstaging prediction have been constructed. The probability nomogram developed by Partin considered PSA, clinical stage, and biopsy GS, but not mpMRI $(8,15)$. This model has been confirmed to overestimate the probability of organ confinement and underestimate the probability of extraprostatic extension (EPE) during application. mpMRI has been considered as an important part of those models for the detailed anatomical information it provides. Feng et al. (6) have demonstrated that adding mpMRI findings to Partin tables and to the MSK nomograms could significantly improve their predictive accuracy, but those results were limited by the relatively small sample study [112]. Martini et al. (9) have developed an mpMRI based nomogram for the prediction of side-specific EPE, including the variables PSA, highest biopsy Gleason grade, and EPE. Despite its relative high AUC (82.11\%), the clinical stage of patients with extracapsular extension on mpMRI should be considered as T3. The clinical stage of patients in those models was determined by digital rectal examination and prostate biopsy, which have been proved to have poor accuracy. Due to the relatively high cost, the adoption rate of mpMRI for staging PCa is low in Europe and the United States. In our cohort all patients underwent mpMRI before biopsy, which would make our model more generalizable.

Our previous studies have confirmed the value of PIRADS in predicting $\mathrm{PCa}$ and clinically significant $\mathrm{PCa}$ in men undergoing repeat prostate biopsy and in predicting pelvic lymph node metastasis at radical prostatectomy $(16,17)$. The aim of this study was to develop a new predictive model incorporating PI-RADS for the prediction of postoperative upstaging. The PSA density, GS, and PIRADS score were found to be independent risk factors for the prediction of upstaging at final pathology. On that basis, we constructed a predictive model based on the cohort from a single institution, in order to predict the probability of upstaging after surgery. With three readily available variables, the combined predictive model performed better than any single factor, with an AUC value of 0.735 $(\mathrm{P}<0.001)$. With high sensitivity and negative predictive value, this model can precisely distinguish organ-confined disease from cases with locally advanced PCa and help in the selection of suitable candidates for nerve sparing surgery.

MpMRI and the PI-RADS v2 criteria $(10,18)$ have improved visualization and characterization of $\mathrm{PCa}$, leading to more accurate diagnosis and treatment. Kozikowski et al. (19) pointed out that with the optimal cutoff value of 5 for predicting side-specific EPE, the sensitivity of the PI-RADS score was twice as high as that of standard MRI staging and the specificity was slightly lower than that of standard MRI staging (83.5\% vs. 92.0\%). Bianchi et al. (20) analyzed 254 patients with low risk PCa and reported that PI-RADS was significantly correlated with upstaging at final pathology. Matsuoka et al. (21) showed that the system may serve as a valuable predictor of extracapsular extension, which may reduce the risk of understaging and facilitate precise preoperative planning.

PSA, a widely used prostate-specific serum marker, may be detected in cases of benign prostate hyperplasia, prostatitis, and $\mathrm{PCa}$. A smaller $\mathrm{PV}$ was proved to be significantly associated with a higher risk of $\mathrm{PCa}$ in prior studies. In the current study, PSA $(\mathrm{OR}, 1.141 ; \mathrm{P}<0.001)$ and PV (OR, 0.980; $\mathrm{P}<0.001)$ were strong predictors for postoperative upstaging. PSA density, the combination of PSA and PV, proved to be one of the most valuable markers to predict upstaging in patients with clinically localized PCa, which is consistent with a previous study (22). However, the predictive value of $\mathrm{f} / \mathrm{t}$ PSA remains controversial in the literature. Aus et al. (23) reported that $\mathrm{f} / \mathrm{t}$ PSA was a predictor of non-organ-confined PCa (stage pT3), whereas other studies reported that $\mathrm{f} / \mathrm{t}$ PSA did not provide additional information about the pathological stage $(24,25)$. Similarly, although age was proved to be associated with aggressive $\mathrm{PCa}$ in our previous study, it was not of predictive value for postoperative upstaging. Systematic biopsy is recommended as the method of choice for patients with elevated PSA or abnormal digital rectal examination in general, whereas there is a certain bias in percentage of positive cores on account of different biopsy strategies, PV, and lesions size. The predictive value of the percentage of positive cores for postoperative upstaging evidently remains unclear. In this study, in the multivariate analysis era, $\mathrm{f} / \mathrm{t}$ PSA and percentage of positive cores were not independent significant predictors of upstaging at final pathology. Thus, these variables were excluded from our final model. 
The present study is not devoid of limitations. First, there is a certain risk of selection bias due to the retrospective nature of our study. Second, the predictive model was constructed on the basis of a sample size that was not very large from a single institution. Third, our model will require external validation in a multicenter study to assess its wider applicability. Forth, due to improvement in data quality over time and the different MRI protocol performed in patients, there might be a certain of difference in the outcome of the MRI.

\section{Conclusions}

PI-RADS assessment was proved to be one of the most valuable predictors for postoperative upstaging in patients with low-intermediate risk PCa. The predictive model on the basis of PI-RADS assessment, PSA density, and biopsy GGG may guide the treatment strategy and select suitable patients for nerve sparing surgery among patients with lowintermediate risk PCa.

\section{Acknowledgments}

Funding: This work was supported by the grant from the Joint Clinical Study of Peking University First Hospital. The authors thank the entire staff of the Department of Urology, Peking University First Hospital.

\section{Footnote}

Conflicts of Interest: All authors have completed the ICMJE uniform disclosure form (available at http://dx.doi. org/10.21037/tau.2020.01.28). GS serves as an unpaid editorial board member of Translational Andrology and Urology from Jun 2019 to May 2021. The other authors have no conflicts of interest to declare.

Ethical Statement: The authors are accountable for all aspects of the work in ensuring that questions related to the accuracy or integrity of any part of the work are appropriately investigated and resolved. Approval was obtained from the institutional review board.

Open Access Statement: This is an Open Access article distributed in accordance with the Creative Commons Attribution-NonCommercial-NoDerivs 4.0 International License (CC BY-NC-ND 4.0), which permits the noncommercial replication and distribution of the article with the strict proviso that no changes or edits are made and the original work is properly cited (including links to both the formal publication through the relevant DOI and the license). See: https://creativecommons.org/licenses/by-nc$\mathrm{nd} / 4.0 /$.

\section{References}

1. Park YH, Kwon OS, Hong SH, et al. Effect of NerveSparing Radical Prostatectomy on Urinary Continence in Patients With Preoperative Erectile Dysfunction. Int Neurourol J 2016;20:69-74.

2. Ficarra V, Novara G, Rosen RC, et al. Systematic review and meta-analysis of studies reporting urinary continence recovery after robot-assisted radical prostatectomy. Eur Urol 2012;62:405-17.

3. Sanda MG, Ronald CC, Crispino T, et al. Clinically Localized Prostate Cancer: AUA/ASTRO/SUO

Guideline. Available online: http://www.auanet.org/ guidelines/clinicallylocalized-prostate-cancer-new-(aua/ astro/suo-guideline-2017)

4. Mottet N, Bellmunt J, Bolla M, et al. EAU-ESTROSIOG Guidelines on Prostate Cancer. Part 1: Screening, Diagnosis, and Local Treatment with Curative Intent. Eur Urol 2017;71:618-29.

5. Alessi S, Pricolo P, Summers P, et al. Low PI-RADS assessment category excludes extraprostatic extension ( $\geq$ pT3a) of prostate cancer: a histology-validated study including 301 operated patients. Eur Radiol 2019;29:5478-87.

6. Feng TS, Sharif-Afshar AR, Wu J, et al. Multiparametric MRI Improves Accuracy of Clinical Nomograms for Predicting Extracapsular Extension of Prostate Cancer. Urology 2015;86:332-7.

7. Curto F, Benijts J, Pansadoro A, et al. Nerve sparing laparoscopic radical prostatectomy: our technique. Eur Urol 2006;49:344-52.

8. Partin AW, Borland RN, Epstein JI, et al. Influence of wide excision of the neurovascular bundle(s) on prognosis in men with clinically localized prostate cancer with established capsular penetration. J Urol 1993;150:142-6; discussion 146-8.

9. Martini A, Gupta A, Lewis SC, et al. Development and internal validation of a side-specific, multiparametric magnetic resonance imaging-based nomogram for the prediction of extracapsular extension of prostate cancer. BJU Int 2018;122:1025-33.

10. Weinreb JC, Barentsz JO, Choyke PL, et al. PI-RADS 
Prostate Imaging - Reporting and Data System: 2015, Version 2. Eur Urol 2016;69:16-40.

11. Mohler JL, Antonarakis ES, Armstrong AJ, et al. Prostate Cancer, Version 2.2019, NCCN Clinical Practice Guidelines in Oncology. J Natl Compr Canc Netw 2019;17:479-505.

12. Epstein JI, Egevad L, Amin MB, et al. The 2014 International Society of Urological Pathology (ISUP) Consensus Conference on Gleason Grading of Prostatic Carcinoma: Definition of Grading Patterns and Proposal for a New Grading System. Am J Surg Pathol 2016;40:244-52.

13. de Rooij M, Hamoen EH, Witjes JA, et al. Accuracy of Magnetic Resonance Imaging for Local Staging of Prostate Cancer: A Diagnostic Meta-analysis. Eur Urol 2016;70:233-45.

14. Hwang I, Lim D, Jeong YB, et al. Upgrading and upstaging of low-risk prostate cancer among Korean patients: a multicenter study. Asian J Androl 2015;17:811-4.

15. Eifler JB, Feng Z, Lin BM, et al. An updated prostate cancer staging nomogram (Partin tables) based on cases from 2006 to 2011. BJU Int 2013;111:22-9.

16. Huang C, Song G, Wang H, et al. MultiParametric Magnetic Resonance Imaging-Based Nomogram for Predicting Prostate Cancer and Clinically Significant Prostate Cancer in Men Undergoing Repeat Prostate Biopsy. Biomed Res Int 2018;2018:6368309.

17. Huang C, Song G, Wang H, et al. Preoperative PIRADS Version 2 scores helps improve accuracy of clinical nomograms for predicting pelvic lymph node metastasis at radical prostatectomy. Prostate Cancer Prostatic Dis 2020;23:116-26.

Cite this article as: Song $G$, Ruan $M$, Wang $H$, Lin Z, Wang X, Li X, Li P, Wang Y, Zhou B, Hu X, Liu H, Wang H, Guo Y. Predictive model using prostate MRI findings can predict candidates for nerve sparing radical prostatectomy among lowintermediate risk prostate cancer patients. Transl Androl Urol 2020;9(2):437-444. doi: 10.21037/tau.2020.01.28
18. Barentsz JO, Richenberg J, Clements R, et al. ESUR prostate MR guidelines 2012. Eur Radiol 2012;22:746-57.

19. Kozikowski M, Zagożdżon B, Gola M, et al. Prostate Imaging Reporting and Data System in prostate cancer staging and planning for radical prostatectomy. Wideochir Inne Tech Maloinwazyjne 2019;14:262-70.

20. Bianchi R, Cozzi G, Petralia G, et al. Multiparametric magnetic resonance imaging and frozen-section analysis efficiently predict upgrading, upstaging, and extraprostatic extension in patients undergoing nerve-sparing roboticassisted radical prostatectomy. Medicine (Baltimore) 2016;95:e4519.

21. Matsuoka Y, Ishioka J, Tanaka H, et al. Impact of the Prostate Imaging Reporting and Data System, Version 2, on MRI Diagnosis for Extracapsular Extension of Prostate Cancer. AJR Am J Roentgenol 2017;209:W76-84.

22. Brassetti A, Lombardo R, Emiliozzi P, et al. Prostatespecific Antigen Density Is a Good Predictor of Upstaging and Upgrading, According to the New Grading System: The Keys We Are Seeking May Be Already in Our Pocket. Urology 2018;111:129-35.

23. Aus G, Becker C, Lilja H, et al. Free-to-total prostatespecific antigen ratio as a predictor of non-organ-confined prostate cancer (stage pT3). Scand J Urol Nephrol 2003;37:466-70.

24. Erdem E, Atsü N, Akbal C, et al. The free-to-total serum prostatic specific antigen ratio as a predictor of the pathological features of prostate cancer. Int Urol Nephrol 2002;34:519-23.

25. Sakai I, Harada K, Hara I, et al. Limited usefulness of the free-to-total prostate-specific antigen ratio for the diagnosis and staging of prostate cancer in Japanese men. Int J Clin Oncol 2004;9:64-7. 